\title{
Neonatal breast abscess
}

\author{
Akshay K. Saxena ${ }^{\mathrm{a}}$, Kushaljit S. Sodhi ${ }^{\mathrm{a}, *}$, Mukesh K. Yadav ${ }^{\mathrm{a}}$, Jai K. Mahajan ${ }^{\mathrm{b}}$ and Niranjan Khandelwal ${ }^{\mathrm{a}}$ \\ ${ }^{a}$ Department of Radiodiagnosis, Postgraduate Institute of Medical Education and Research, Chandigarh, India \\ ${ }^{\mathrm{b}}$ Department of Pediatric Surgery, Postgraduate Institute of Medical Education and Research, Chandigarh, India
}

\begin{abstract}
Neonatal mastitis and abscess are an uncommon entity with an uncertain mechanism of onset. Ultrasonography can be useful in detecting early stage of abscess formation and hence in expedition of definitive treatment, as it was proved in our case, in which a diagnosis of neonatal breast abscess was made based on ultrasound examination of breast.
\end{abstract}

Keywords: Neonatal mastitis, ultrasonography

\section{Introduction}

Neonatal mastitis and abscess are an uncommon condition, with only one to two reported cases per year from large medical centers [1]. Usually, the infection remains confined to the breast. However, it has the potential to cause fasciitis, cellulitis, osteomyelitis and generalized sepsis [1-3]. Hence, this entity needs to be diagnosed early and treated promptly to prevent the development of localized complications or sepsis.

We report a recent case of neonatal breast abscess, which was successfully treated with combined surgical and medical management.

\section{Case report}

An appropriately immunized, a 21-day-old, full term female neonate, developed a small nodular swelling in the left mammary area, for the last 7 days. Her systemic examination was unremarkable. On examination, she had approximately $2 \times 2 \mathrm{~cm}$ size swelling in the left retroareolar region, which was soft on palpation, with positive signs of inflammation, i.e. increased local temperature, redness of skin and tenderness. No associated lymphadenopathy was seen. Right mammary area was normal. Clinical diagnosis of physiological hypertrophy of left breast was made. Ultrasound examination

\footnotetext{
*Corresponding author: Kushaljit S. Sodhi, Department of Radiodiagnosis, PGIMER, Chandigarh, India. Tel.: +91 172 2756380; Fax: +91 172 2744401; E-mail: sodhiks@gmail.com.
}

revealed heterogeneously hypoechoic area in left nipple region with few anechoic components within it (Fig. 1). A radiological diagnosis of abscess formation was suggested. A follow up ultrasound examination performed 2 days later revealed increase in size as well as increase in the necrotic component of the lesion.

Incision and drainage was performed which yielded approximately $3 \mathrm{~mL}$ of thick pus, which grew Staphylococcus aureus on culture. It was resistant to methicillin/ oxacillin and cefotaxime but sensitive to amoxicillin and clavulinic acid combination (which were already started orally). The baby responded well and showed clinical improvement with this combined surgical and medical management.

\section{Discussion}

Physiological hypertrophy of breast is well known in neonates. Out of many prevalent theories, perhaps the most widely accepted pathogenesis of neonatal mastitis, involves a maternal hormonally stimulated neonatal breast allowing pathogens from mucous membrane and skin to reach breast via mammary ducts and nipple [1,2].

Neonatal mastitis is most commonly seen at 3rd to 5 th week of life, with a peak at 3 week of life. It is more common in females [1]. The mechanism of onset remains still remains uncertain Most commonly implicated organism is $S$. aureus. However, other organisms 


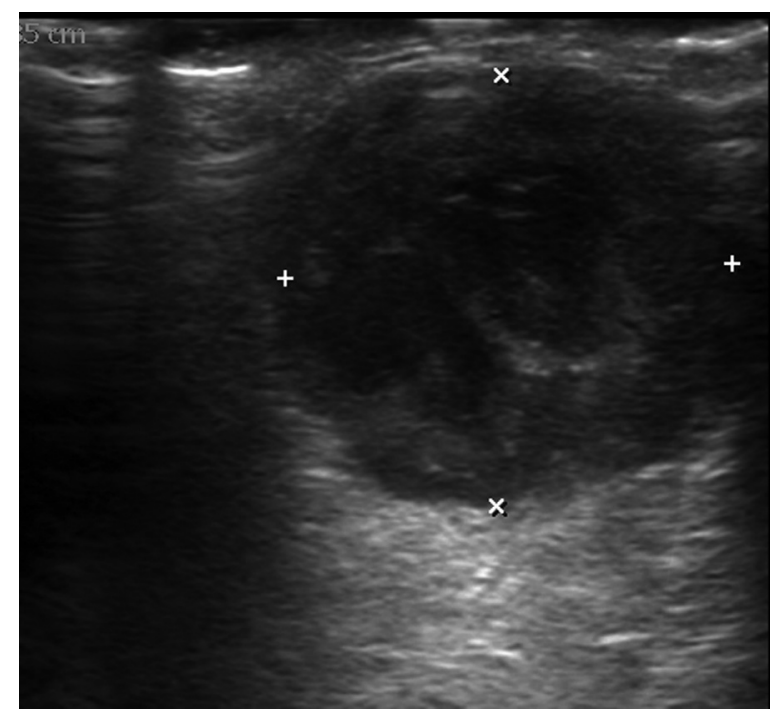

Fig. 1. Ultrasound of left mammary region in the transverse plane reveals an abscess formation.

including Staphylococcus epidermidis, Streptococcus, and Escherichia coli have also been implicated [1-3].

Breast abscess may be diagnosed clinically if fluctuance is present. Ultrasound is advocated in children in whom either a developing abscess is suspected, or fluctuance is only equivocally present, poor response to antibiotics and if mastitis itself is questionable. Ultrasonography can be used to detect the abscess formation early in the course of disease and subsequently early institution of surgical management (incision and drainage) combined with medical management. As seen in our indexed case, the methicillin resistant $S$. aureus has been described as a cause of neonatal breast abscess in a post partum female as well as her infant [4]. In the newborn period, intramuscular and oral use of antibiotics is not recommended because of relatively poor absorption. Intravenous administration is considered as a safe route of antibiotic treatment in infection in neonatal period. Hence, in our indexed case, the drainage was more important in the neonatal breast abscess treatment.

Our case highlights the importance of ultrasound in early diagnosis and prompt management (drainage) of the abscess, which helps to prevent the development of local complications and generalized sepsis, besides future problems of breast development and function.

\section{References}

[1] Borders H, Mychaliska G, Gebarski KS. Sonographic features of neonatal mastitis and breast abscess. Pediatr Radiol 2009; 39: 955-8.

[2] Faden H. Mastitis in children from birth to 17 years. Pediatr Infect Dis J 2005; 24: 1113.

[3] Efrat M, Mogilner JG, Iujtman M, Eldemberg D, Kunin J, Eldar S. Neonatal mastitis-diagnosis and treatment. Isr $\mathrm{J}$ Med Sci 1995; 31: 558-60.

[4] Montalto M, Lui B. MRSA as a cause of postpartum breast abscess in infant and mother. J Hum Lact 2009; 25: 448-50. 